Mathematical Research Letters 1, 769-796 (1994)

\title{
MONOPOLES AND FOUR-MANIFOLDS
}

\author{
EDWARD WITTEN
}

\begin{abstract}
A вsтract. Recent developments in the understanding of $N=2$ supersymmetric Yang-Mills theory in four dimensions suggest a new point of view about Donaldson theory of four manifolds: instead of defining fourmanifold invariants by counting $S U(2)$ instantons, one can define equivalent four-manifold invariants by counting solutions of a non-linear equation with an abelian gauge group. This is a "dual" equation in which the gauge group is the dual of the maximal torus of $S U(2)$. The new viewpoint suggests many new results about the Donaldson invariants.
\end{abstract}

\section{Introduction}

For some years now it has been known that Donaldson theory is equivalent to a quantum field theory, in fact, a twisted version of $N=2$ supersymmetric Yang-Mills theory [1]. The question therefore arises of whether this viewpoint is actually useful for computing Donaldson invariants [2] or understanding their properties.

One standard physical technique is to cut and sum over physical states. In the context of Donaldson theory, such methods have been extensively developed by mathematicians, starting with the work of Floer [3]. So far, despite substantial efforts, the physical reformulation has not given any essentially new insight about these methods.

Another approach to using physics to illuminate Donaldson theory starts with the fact that the $N=2$ gauge theory is asymptotically free; therefore, it is weakly coupled in the ultraviolet and strongly coupled in the infrared. Since the Donaldson invariants - that is, the correlation functions of the twisted theory - are metric independent, they can be computed in the ultraviolet or the infrared, as one wishes. Indeed, the weak coupling in the ultraviolet is used to show that the quantum field theory correlation functions do coincide with the Donaldson invariants.

If one could understand the infrared behavior of the $N=2$ theory, one might get a quite different description and, perhaps, a quite different way to

Received November 14, 1994. 
compute the Donaldson invariants. Until recently, this line of thought was rather hypothetical for general four-manifolds since the infrared behavior of $N=2$ super Yang-Mills theory in the strong coupling region was unknown. Previous work along these lines was therefore limited to Kähler manifolds, where one can reduce the discussion to the $N=1$ theory, whose infrared behavior was known. This led to an almost complete determination [4] of the Donaldson invariants of Kähler manifolds with $H^{2,0} \neq 0$.

The purpose of the present paper is to exploit recent work by Seiberg and the author $[5,6]$ in which the infrared behavior of the $N=2$ theory was determined using methods somewhat akin to methods that have shed light on various $N=1$ theories (for a survey see [7]). The answer turned out to be quite surprising: the infrared limit of the $N=2$ theory in the "strongly coupled" region of field space is equivalent to a weakly coupled theory of abelian gauge fields coupled to "monopoles." The monopole theory is dual to the original theory in the sense that, for instance, the gauge group is the dual of the maximal torus of the original gauge group.

Since the dual theory is weakly coupled in the infrared, everything is computable in that region, and in particular for gauge group $S U(2)$, one does get an alternative formulation of the usual Donaldson invariants. Instead of computing the Donaldson invariants by counting $S U(2)$ instanton solutions, one can obtain the same invariants by counting the solutions of the dual equations, which involve $U(1)$ gauge fields and monopoles. ${ }^{1}$

This formulation makes manifest various properties of the Donaldson invariants. For instance, one can get new proofs of some of the classic results of Donaldson theory; one gets a new description of the basic classes of Kronheimer and Mrowka [8,9], and some new results about them; one gets a new understanding of the "simple type" condition for four-manifolds; one finds new types of vanishing theorems that severely limit the behavior of Donaldson theory on manifolds that admit a metric of positive scalar curvature; and one gets a complete determination of the Donaldson invariants of Kähler manifolds with $H^{2,0} \neq 0$, eliminating the assumptions made in [4] about the canonical divisor.

It should be possible to justify directly the claims sketched in this paper about the consequences of the monopole equations even if the relation to

\footnotetext{
${ }^{1}$ In this paper, we only consider Donaldson theory with gauge group $S U(2)$ or $S O(3)$, but an analogous dual description by abelian gauge fields and monopoles will hold for any compact Lie group, the gauge group of the dual theory being always the dual of the maximal torus of the original gauge group. For example, most of the results needed to write the precise monopole equations for $S U(N)$ have been obtained recently [10,11].
} 
Donaldson theory is difficult to prove. The reformulation may make the problems look quite different as the gauge group is abelian and the most relevant moduli spaces are zero dimensional. From a physical point of view the dual description via monopoles and abelian gauge fields should be simpler than the microscopic $S U(2)$ description since in the renormalization group sense it arises by "integrating out the irrelevant degrees of freedom."

The monopole equations are close cousins of equations studied in section two of [12]; the reason for the analogy is that in each case one is studying $N=2$ theories of hypermultiplets coupled to vector multiplets. The investigation in [12] dealt with microscopic Montonen-Olive duality [13,14,15], while the duality in Donaldson theory $[5,6]$ is a sort of phenomenological analog of this.

The monopole equations, definition of four-manifold invariants, and relation to Donaldson theory are stated in section two of this paper. Vanishing theorems are used in section three to deduce some basic properties. Invariants of Kähler manifolds are computed in section four. A very brief sketch of the origin in physics is in section five. A fuller account of the contents of section five will appear elsewhere [16].

\section{The Monopole Equations}

Let $X$ be an oriented, closed four-manifold on which we pick a Riemannian structure with metric tensor $g . \Lambda^{p} T^{*} X$, or simply $\Lambda^{p}$, will denote the bundle of real-valued $p$-forms, and $\Lambda^{2, \pm}$ will be the sub-bundle of $\Lambda^{2}$ consisting of self-dual or anti-self-dual forms.

The monopole equations relevant to $S U(2)$ or $S O(3)$ Donaldson theory can be described as follows. If $w_{2}(X)=0$, then $X$ is a spin manifold and one can pick positive and negative spin bundles $S^{+}$and $S^{-}$, of rank two. (If there is more than one spin structure, the choice of a spin structure will not matter as we will ultimately sum over twistings by line bundles.) In that case, introduce a complex line bundle $L$; the data in the monopole equation will be a connection $A$ on $L$ and a section $M$ of $S^{+} \otimes L$. The curvature two-form of $A$ will be called $F$ or $F(A)$; its self-dual and anti-self-dual projections will be called $F^{+}$and $F^{-}$.

If $X$ is not spin, the $S^{ \pm}$do not exist, but their projectivizations $\mathbf{P} S^{ \pm}$ do exist (as bundles with fiber isomorphic to $\mathbf{C P}^{1}$ ). A $\operatorname{Spin}_{c}$ structure (which exists on any oriented four-manifold [17]) can be described as a choice of a rank two complex vector bundle, which we write as $S^{+} \otimes L$, whose projectivization is isomorphic to $\mathbf{P} S^{+}$. In this situation, $L$ does not 
exist as a line bundle, but $L^{2}$ does $^{2}$; the motivation for writing the $\operatorname{Spin}_{c}$ bundle as $S^{+} \otimes L$ is that the tensor powers of this bundle obey isomorphisms suggested by the notation. For instance, $\left(S^{+} \otimes L\right)^{\otimes 2} \cong L^{2} \otimes\left(\Lambda^{0} \oplus \Lambda^{2,+}\right)$. The data of the monopole equation are now a section $M$ of $S^{+} \otimes L$ and a connection on $S^{+} \otimes L$ that projects to the Riemannian connection on $\mathbf{P} S^{+}$. The symbol $F(A)$ will now denote $1 / 2$ the trace of the curvature form of $S^{+} \otimes L$.

Since $L^{2}$ is an ordinary line bundle, one has an integral cohomology class $x=-c_{1}\left(L^{2}\right) \in H^{2}(X, \mathbf{Z})$. (The minus sign makes some later formulas come out in a standard form.) Note that $x$ reduces modulo two to $w_{2}(X)$; in particular, if $w_{2}(X)=0$, then $L$ exists as a line bundle and $x=-2 c_{1}(L)$.

To write the monopole equations, recall that $S^{+}$is symplectic or pseudoreal, so that if $M$ is a section of $S^{+} \otimes L$, then the complex conjugate $\bar{M}$ is a section of $S^{+} \otimes L^{-1}$. The product $M \otimes \bar{M}$ would naturally lie in $\left(S^{+} \otimes L\right) \otimes\left(S^{+} \otimes L^{-1}\right) \cong \Lambda^{0} \oplus \Lambda^{2,+} . F^{+}$also takes values in $\Lambda^{2,+}$ making it possible to write the following equations. Introduce Clifford matrices $\Gamma_{i}$ (with anticommutators $\left\{\Gamma_{i}, \Gamma_{j}\right\}=2 g_{i j}$ ), and set $\Gamma_{i j}=\frac{1}{2}\left[\Gamma_{i}, \Gamma_{j}\right]$. Then the equations are $^{3}$

$$
\begin{aligned}
F_{i j}^{+} & =-\frac{i}{2} \bar{M} \Gamma_{i j} M \\
\sum_{i} \Gamma^{i} D_{i} M & =0 .
\end{aligned}
$$

In the second equation, $\sum_{i} \Gamma^{i} D_{i}$ is the Dirac operator $D$ that maps sections of $S^{+} \otimes L$ to sections of $S^{-} \otimes L$. We will sometimes abbreviate the first as $F^{+}=(M \bar{M})^{+}$. Alternatively, if positive spinor indices are written $A, B, C$, and negative spinor indices as $A^{\prime}, B^{\prime}, C^{\prime},{ }^{4}$ the equations can be written

$$
\begin{aligned}
F_{A B} & =\frac{i}{2}\left(M_{A} \bar{M}_{B}+M_{B} \bar{M}_{A}\right) \\
D_{A A^{\prime}} M^{A} & =0 .
\end{aligned}
$$

\footnotetext{
${ }^{2}$ One might be tempted to call this bundle $L$ and write the $\operatorname{Spin}_{c}$ bundle as $S^{+} \otimes L^{1 / 2}$; that amounts to assigning magnetic charge $1 / 2$ to the monopole and seems unnatural physically.

${ }^{3}$ To physicists the connection form $A$ on a unitary line bundle is real; the covariant derivative is $d_{A}=d+i A$ and the curvature is $F=d A$ or in components $F_{i j}=\partial_{i} A_{j}-$ $\partial_{j} A_{i}$.

${ }^{4}$ Spinor indices are raised and lowered using the invariant tensor in $\Lambda^{2} S^{+}$. In components, if $M^{A}=\left(M^{1}, M^{2}\right)$, then $M_{A}=\left(-M_{2}, M_{1}\right)$. One uses the same operation in interpreting $\bar{M}$ as a section of $S^{+} \otimes L$, so $\bar{M}^{A}=\left(\bar{M}^{2},-\bar{M}^{1}\right)$. Also $F_{A B}=\frac{1}{4} F_{i j} \Gamma_{A B}^{i j}$.
} 
As a first step in understanding these equations, let us work out the virtual dimension of the moduli space $\mathcal{M}$ of solutions of the equations up to gauge transformation. The linearization of the monopole equations fits into an elliptic complex

$$
0 \rightarrow \Lambda^{0} \stackrel{s}{\longrightarrow} \Lambda^{1} \oplus\left(S^{+} \otimes L\right) \stackrel{t}{\longrightarrow} \Lambda^{2,+} \oplus\left(S^{-} \otimes L\right) \rightarrow 0 .
$$

Here $t$ is the linearization of the monopole equations, and $s$ is the map from zero forms to deformations in $A, M$ given by the infinitesimal action of the gauge group. Since we wish to work with real operators and determine the real dimension of $\mathcal{M}$, we temporarily think of $S^{ \pm} \otimes L$ as real vector bundles (of rank four). Then an elliptic operator

$$
T: \Lambda^{1} \oplus\left(S^{+} \otimes L\right) \rightarrow \Lambda^{0} \oplus \Lambda^{2,+} \oplus\left(S^{-} \otimes L\right)
$$

is defined by $T=s^{*} \oplus t$. The virtual dimension of the moduli space is given by the index of $T$. By dropping terms in $T$ of order zero, $T$ can be deformed to the direct sum of the operator ${ }^{5} d+d^{*}$ from $\Lambda^{1}$ to $\Lambda^{0} \oplus \Lambda^{2,+}$ and the Dirac operator from $S^{+} \otimes L$ to $S^{-} \otimes L$. The index of $T$ is the index of $d+d^{*}$ plus twice what is usually called the index of the Dirac operator; the factor of two comes from looking at $S^{ \pm} \otimes L$ as real bundles of twice the dimension. Let $\chi$ and $\sigma$ be the Euler characteristic and signature of $X$. Then the index of $d+d^{*}$ is $-(\chi+\sigma) / 2$, while twice the Dirac index is $-\sigma / 4+c_{1}(L)^{2}$. The virtual dimension of the moduli space is the sum of these or

$$
W=-\frac{2 \chi+3 \sigma}{4}+c_{1}(L)^{2}
$$

When this number is negative, there are generically no solutions of the monopole equations. When $W=0$, that is, when $x=-c_{1}\left(L^{2}\right)=-2 c_{1}(L)$ obeys

$$
x^{2}=2 \chi+3 \sigma
$$

then the virtual dimension is zero and the moduli space generically consists of a finite set of points $P_{i, x}, i=1 \ldots t_{x}$. With each such point, one can associate a sign $\epsilon_{i, x}= \pm 1$ - the sign of the determinant of $T$ as we discuss

\footnotetext{
${ }^{5}$ What is meant here is of course a projection of the $d+d^{*}$ operator to self-dual forms.
} 
momentarily. Once this is done, define for each $x$ obeying (2.6) an integer $n_{x}$ by

$$
n_{x}=\sum_{i} \epsilon_{i, x}
$$

We will see later that $n_{x}=0$-indeed, the moduli space is empty-for all but finitely many $x$. Under certain conditions that we will discuss in a moment, the $n_{x}$ are topological invariants.

Note that $W=0$ if and only if the index of the Dirac operator is

$$
\Delta=\frac{\chi+\sigma}{4}
$$

In particular, $\Delta$ must be an integer to have non-trivial $n_{x}$. Similarly, $\Delta$ must be integral for the Donaldson invariants to be non-trivial (otherwise $S U(2)$ instanton moduli space is odd dimensional).

For the sign of the determinant of $T$ to make sense, one must trivialize the determinant line of $T$. This can be done by deforming $T$ as above to the direct sum of $d+d^{*}$ and the Dirac operator. If the Dirac operator, which naturally has a non-trivial complex determinant line, is regarded as a real operator, then its determinant line is naturally trivial (as a complex line has a natural orientation). The $d+d^{*}$ operator is independent of $A$ and $M$ (as the gauge group is abelian), and its determinant line is trivialized once and for all by picking an orientation of $H^{1}(X, \mathbf{R}) \oplus H^{2,+}(X, \mathbf{R})$. Note that this is the same data needed by Donaldson [18] to orient instanton moduli spaces for $S U(2)$; this is an aspect of the relation between the two theories.

If one replaces $L$ by $L^{-1}, A$ by $-A$, and $M$ by $\bar{M}$, the monopole equations are invariant, but the trivialization of the determinant line is multiplied by $(-1)^{\delta}$ with $\delta$ the Dirac index. Hence the invariants for $L$ and $L^{-1}$ are related by

$$
n_{-x}=(-1)^{\Delta} n_{x}
$$

For $W<0$, the moduli space is generically empty. For $W>0$ one can try, as in Donaldson theory, to define topological invariants that involve integration over the moduli space. Donaldson theory does not detect those invariants in known situations. We will see in section three that even when $W>0$, the moduli space is empty for almost all $x$. 


\section{Topological Invariance}

In general, the number of solutions of a system of equations weighted by the sign of the determinant of the operator analogous to $T$ is always a topological invariant if a suitable compactness holds. If as in the case at hand one has a gauge invariant system of equations, and one wishes to count gauge orbits of solutions up to gauge transformations, then one requires (i) compactness; and (ii) free action of the gauge group on the space of solutions.

Compactness fails if a field or its derivatives can go to infinity. The Weitzenbock formula used in section three to discuss vanishing theorems indicates that these phenomena do not occur for the monopole equations. To explain the contrast with Donaldson theory, note that for $S U(2)$ instantons, compactness fails precisely [19] because an instanton can shrink to zero size. This is possible because (i) the equations are conformally invariant, (ii) they have non-trivial solutions on a flat $\mathbf{R}^{4}$, and (iii) embedding such a solution, scaled to very small size, on any four-manifold gives a highly localized approximate solution of the instanton equations (which can sometimes [20] be perturbed to an exact solution). The monopole equations by contrast are scale invariant but (as follows immediately from the Weitzenbock formula) they have no non-constant $L^{2}$ solutions on flat $\mathbf{R}^{4}$ (or after dimensional reduction on flat $\mathbf{R}^{n}$ with $1 \leq n \leq 3$ ). So there is no analog for the monopole equations of the phenomenon where an instanton shrinks to zero size.

On the other hand, an obstruction does arise, just as in Donaldson theory (in what follows we imitate some arguments in [21]) from the question of whether the gauge group acts freely on the space of solutions of the monopole equations. The only way for the gauge group to fail to act freely is that there might be a solution with $M=0$, in which case a constant gauge transformation acts trivially. A solution with $M=0$ necessarily has $F^{+}=0$, that is, it is an abelian instanton.

Since $F / 2 \pi$ represents the first Chern class of the line bundle $L$, it is integral; in particular if $F^{+}=0$ then $F / 2 \pi$ lies in the intersection of the integral lattice in $H^{2}(X, \mathbf{R})$ with the anti-self-dual subspace $H^{2,-}(X, \mathbf{R})$. As long as $b_{2}^{+} \geq 1$, so that the self-dual part of $H^{2}(X, \mathbf{R})$ is non-empty, the intersection of the anti-self-dual part and the integral lattice generically consists only of the zero vector. In this case, for a generic metric on $X$, there are no abelian instantons (except for $x=0$, which we momentarily exclude) and $n_{x}$ is well-defined. 
To show that the $n_{x}$ are topological invariants, one must further show that any two generic metrics on $X$ can be joined by a path along which there is never an abelian instanton. As in Donaldson theory, this can fail if $b_{2}^{+}=1$. In that case, the self-dual part of $H^{2}(X, \mathbf{R})$ is one dimensional, and in a generic one parameter family of metrics on $X$, one may meet a metric for which there is an abelian instanton. When this occurs, the $n_{x}$ can jump. Let us analyze how this happens, assuming for simplicity that $b_{1}=0$. Given $b_{1}=0$ and $b_{2}{ }^{+}=1$, one has $W=0$ precisely if the index of the Dirac equation is 1 . Therefore, there is generically a single solution $M_{0}$ of the Dirac equation $D M=0$.

The equation $F^{+}(A)=0$ cannot be obeyed for a generic metric on $X$, but we want to look at the behavior near a special metric for which it does have a solution. Consider a one parameter family of metrics parametrized by a real parameter $\epsilon$, such that at $\epsilon=0$ the self-dual subspace in $H^{2}(X, \mathbf{R})$ crosses a "wall" and a solution $A_{0}$ of $F^{+}(A)=0$ appears. Hence for $\epsilon=0$, we can solve the monopole equations with $A=A_{0}, M=0$. Let us see what happens to this solution when $\epsilon$ is very small but non-zero. We set $M=m M_{0}$, with $m$ a complex number, to obey $D M=0$, and we write $A=A_{0}+\epsilon \delta A$. The equation $F^{+}(A)-(M \bar{M})^{+}=0$ becomes

$$
F^{+}\left(A_{0}\right)+(d \delta A)^{+}-|m|^{2}\left(M_{0} \bar{M}_{0}\right)^{+}=0 .
$$

As the cokernel of $A \rightarrow F^{+}(A)$ is one dimensional, $\delta A$ can be chosen to project the left hand side of equation (2.10) into a one dimensional subspace. (As $b_{1}=0$, this can be done in a unique way up to a gauge transformation.) The remaining equation looks near $\epsilon=0$ like

$$
c \epsilon-m \bar{m}=0
$$

with $c$ a constant. The $\epsilon$ term on the left comes from the fact that $F^{+}\left(A_{0}\right)$ is proportional to $\epsilon$.

Now we can see what happens for $\epsilon \neq 0$ to the solution that at $\epsilon=0$ has $A=A_{0}, M=M_{0}$. Depending on the sign of $c$, there is a solution for $m$, uniquely determined up to gauge transformation, for $\epsilon>0$ and no solution for $\epsilon<0$, or vice-versa. Therefore $n_{x}$ jumps by \pm 1 , depending on the sign of $c$, in passing through $\epsilon=0$. To compare this precisely to the similar behavior of Donaldson theory, one would also need to understand the role of the $u$ plane, discussed in section five. 
The trivial abelian instanton with $x=0$ is an exception to the above discussion, since it cannot be removed by perturbing the metric. To define $n_{0}$, perturb the equation $F_{A B}=\frac{i}{2}\left(M_{A} \bar{M}_{B}+M_{B} \bar{M}_{A}\right)$ to

$$
F_{A B}=\frac{i}{2}\left(M_{A} \bar{M}_{B}+M_{B} \bar{M}_{A}\right)-p_{A B},
$$

with $p$ a self-dual harmonic two-form; with this perturbation, the gauge group acts freely on the solution space. Then define $n_{0}$ as the number of gauge orbits of solutions of the perturbed equations weighted by sign in the usual way. This is invariant under continuous deformations of $p$ for $p \neq 0$; as long as $b_{2}^{+}>1$, so that the space of possible $p$ 's is connected, the integer $n_{0}$ defined this way is a topological invariant.

The perturbation just pointed out will be used later in the case that $p$ is the real part of a holomorphic two-form to compute the invariants of Kähler manifolds with $b_{2}^{+}>1$. It probably has other applications; for instance, the case that $p$ is a symplectic form is of interest.

\section{Relation To Donaldson Theory}

With an appropriate restriction on $b_{2}^{+}$, the $n_{x}$ have (by an argument sketched in section five) a relation to the Donaldson invariants that will now be stated.

Let us recall that in $S U(2)$ Donaldson theory, one wishes to compute the integrals or expectation values of certain cohomology classes or quantum field operators: for every Riemann surface (or more generally every twodimensional homology cycle) $\Sigma$ in $X$, one has an operator $I(\Sigma)$ of dimension (or $R$ charge or ghost number) two; there is one additional operator $\mathcal{O}$, of dimension four. For every value of the instanton number, one computes the expectation value of a suitable product of these operators by integration over instanton moduli space using a recipe due to Donaldson, or by evaluating a suitable quantum field theory correlation function as in [1]. It is natural to organize this data in the form of a generating function

$$
\left\langle\exp \left(\sum_{a} \alpha_{a} I\left(\Sigma_{a}\right)+\lambda \mathcal{O}\right)\right\rangle,
$$

summed over instanton numbers; here the $\Sigma_{a}$ range over a basis of $H_{2}(X, \mathbf{R})$ and $\lambda, \alpha_{a}$ are complex numbers.

Let $v=\sum_{a} \alpha_{a}\left[\Sigma_{a}\right]$, with $\left[\Sigma_{a}\right]$ the cohomology class that is Poincaré dual to $\Sigma_{a}$. So for instance $v^{2}=\sum_{a, b} \alpha_{a} \alpha_{b} \Sigma_{a} \cdot \Sigma_{b}$ (here $\Sigma_{a} \cdot \Sigma_{b}$ is the intersection 
number of $\Sigma_{a}$ and $\left.\Sigma_{b}\right)$, and for any $x \in H^{2}(X, \mathbf{Z}), v \cdot x=\sum_{a} \alpha_{a}\left(\Sigma_{a}, x\right)$. Let as before $\Delta=(\chi+\sigma) / 4$.

A four-manifold is said to be of simple type if the generating function in (2.13) is annihilated by $\partial^{2} / \partial \lambda^{2}-4$; all known simply-connected fourmanifolds with $b_{2}^{+}>1$ have this property. The relation of the simple type condition to physics is discussed in section five. I claim that for manifolds of simple type

$$
\begin{array}{r}
\left\langle\exp \left(\sum_{a} \alpha_{a} I\left(\Sigma_{a}\right)+\lambda \mathcal{O}\right)\right\rangle=2^{1+\frac{1}{4}(7 \chi+11 \sigma)}\left(\exp \left(\frac{v^{2}}{2}+2 \lambda\right) \sum_{x} n_{x} e^{v \cdot x}\right. \\
\left.+i^{\Delta} \exp \left(-\frac{v^{2}}{2}-2 \lambda\right) \sum_{x} n_{x} e^{-i v \cdot x}\right) .
\end{array}
$$

That the expression is real follows from (2.9).

As sketched in section five, this formula is a sort of corollary of the analysis of $N=2$ supersymmetric Yang-Mills theory in $[5,6]$. Here I will just make a few remarks:

(1) The structure in (2.14) agrees with the general form proved by Kronheimer and Mrowka $[8,9]$. The classes $x \in H^{2}(X, \mathbf{Z})$ such that $n_{x} \neq 0$ are the basic classes in their terminology. From the properties by which $x$ and $n_{x}$ were defined, we have that $x$ is congruent to $w_{2}(X)$ modulo 2 and that $x^{2}=2 \chi+3 \sigma$. The first assertion is a result of Kronheimer and Mrowka and the second was conjectured by them.

(2) The prefactor $2^{1+\frac{1}{4}(7 \chi+11 \sigma)}$ has the following origin, as in [4]. One factor of two comes because, even though the center of $S U(2)$ acts trivially on the $S U(2)$ instanton moduli space, the Donaldson invariants are usually defined without dividing by two. The remaining factor of $2^{\frac{1}{4}(7 \chi+11 \sigma)}$ is a $c$-number renormalization factor that arises in comparing the microscopic $S U(2)$ theory to the dual description with monopoles. (In [16] a more general function of the form $e^{a(u) \chi+b(u) \sigma}$ that arises on the complex $u$ plane will be calculated.) Some coefficients in the formula such as the $7 / 4$ and $11 / 4$ were fixed in [4] to agree with calculations of special cases of Donaldson invariants.

(3) Most fundamentally, in the above formula, the first term, that is

$$
\exp \left(\frac{v^{2}}{2}+2 \lambda\right) \sum_{x} n_{x} e^{v \cdot x}
$$


is the contribution from one vacuum at $u=\Lambda^{2}$, and the second term,

$$
i^{\Delta} \exp \left(-\frac{v^{2}}{2}-2 \lambda\right) \sum_{x} n_{x} e^{-i v \cdot x},
$$

is the contribution of a second vacuum at $u=-\Lambda^{2}$. These terms are analogous to the two terms in equation (2.66) of [4]. The factor of $i^{\Delta}$ arises, as there, because of a global anomaly in the discrete symmetry that exchanges the two vacua. This factor can be written in the form $e^{a \chi+b \sigma}$ and so means that the two vacua have different values of the renormalization mentioned in the last paragraph. The replacement of $e^{v \cdot x}$ in the first vacuum by $e^{-i v \cdot x}$ in the second is likewise determined by the symmetries, as in [4], and can be seen microscopically. For a general simple compact gauge group, the analogous sum will have $h$ terms ( $h$ the dual Coxeter number) associated with $h$ vacua.

(4) This formula generalizes as follows for the case that the gauge group is $S O(3)$ rather than $S U(2)$. Consider an $S O(3)$ bundle $E$ with, say, $w_{2}(E)=z$. Define a generating functional of correlation functions summed over bundles with all values of the first Pontryagin class but fixed $w_{2}$. Pick an integral lift of $w_{2}(X)$, and, using the fact that the $x$ 's are congruent to $w_{2}(X) \bmod$ two, let $x^{\prime}$ be such that $2 x^{\prime}=x+w_{2}(X)$. Then $w_{2}(E) \neq 0$ modifies the derivation of (2.14) only by certain minus signs that appear in the duality transformation that relates the microscopic and macroscopic descriptions; the result is

$$
\begin{aligned}
&\left\langle\exp \left(\sum_{a} \alpha_{a} I\left(\Sigma_{a}\right)+\lambda \mathcal{O}\right)\right\rangle_{z} \\
&=2^{1+\frac{1}{4}(7 \chi+11 \sigma)}\left(\exp \left(\frac{v^{2}}{2}+2 \lambda\right) \sum_{x}(-1)^{x^{\prime} \cdot z} n_{x} e^{v \cdot x}\right. \\
&\left.+i^{\Delta-z^{2}} \exp \left(-\frac{v^{2}}{2}-2 \lambda\right) \sum_{x}(-1)^{x^{\prime} \cdot z} n_{x} e^{-i v \cdot x}\right) .
\end{aligned}
$$

The replacement of $i^{\Delta}$ by $i^{\Delta-z^{2}}$ arises, as in equation (2.79) of [4] (where $w_{2}(E)$ is written as $\left.x\right)$, because the global anomaly has an extra term that depends on $z$. (Note that as $z$ is defined modulo two, $z^{2}$ is well-defined modulo four.) The factor of $(-1)^{x^{\prime} \cdot z}$ was obtained in $[8,9]$ for manifolds of simple type and in [4] for Kähler manifolds. If the integral lift of $w_{2}(X)$ used 
in defining $x^{\prime}$ is shifted by $w_{2}(X) \rightarrow w_{2}(X)+2 y$, then (2.17) is multiplied by $(-1)^{y \cdot z}$. The reason for this factor is that (2.17) is reproducing the conventional Donaldson invariants, whose sign depends on the orientation of the instanton moduli spaces. A natural orientation [18] depends on an integral lift of $w_{2}(X)$ and transforms as (2.17) does if this lift is changed.

(5) For Kähler manifolds with $b_{2}^{+}>1$, the quantities entering in (2.14) will be completely computed in section four. We will find that, letting $\eta$ be a holomorphic two-form, the sum in (2.14) can be interpreted as a sum over factorizations $\eta=\alpha \beta$ with $\alpha$ and $\beta$ holomorphic sections of $K^{1 / 2} \otimes L^{ \pm 1}$. Each such factorization contributes \pm 1 to $n_{x}$ with $x=-2 c_{1}(L)$ provided $x^{2}=c_{1}(K)^{2}$; the contribution is +1 or -1 according to a formula computed at the end of section four.

\section{Imitating Arguments From Donaldson Theory}

Apart from relating Donaldson theory to the monopole equations, one can simply try to adapt familiar arguments about Donaldson theory to the monopole equations. We have already seen some examples.

As another example, consider Donaldson's theorem [2] asserting that the Donaldson invariants vanish for a connected sum $X \# Y$ of four-manifolds $X$ and $Y$ which each have $b_{2}{ }^{+}>0$. The theorem is proved by considering a metric on $X \# Y$ in which $X$ and $Y$ are joined by a long neck of the form $\mathbf{S}^{3} \times I$, with $I$ an interval in $\mathbf{R}$. Take the metric on the neck to be the product of the standard metric on $\mathbf{S}^{3}$ and a metric that assigns length $t$ to $I$, and consider the monopole equations on this space. For $t \rightarrow \infty$, any solution of the monopole equations will vanish in the neck because of the positive scalar curvature of $\mathbf{S}^{3}$ (this follows from the Weitzenbock formula of the next section). This lets one define a $U(1)$ action on the moduli space $\mathcal{M}$ (analogous to the $S O(3)$ action used by Donaldson) by gauge transforming the solutions on $Y$ by a constant gauge transformation, leaving fixed the data on $X$. A fixed point of this $U(1)$ action would be a solution for which $M$ vanishes on $X$ or on $Y$. But as $X$ and $Y$ both have $b_{2}{ }^{+}>0$, there is no such solution if generic metrics are used on the two sides. A zero dimensional moduli space with a free $U(1)$ action is empty, so the basic invariants would be zero for such connected sums. (A free $U(1)$ action also leads to vanishing of the higher invariants.) Since we will see in section four that the invariants are non-zero for Kähler manifolds (analogous to another basic result of Donaldson), one gets a proof directly from the monopole equations and independent of the equivalence to Donaldson theory that 
algebraic surfaces do not have connected sum decompositions with $b_{2}^{+}>0$ on both sides.

If one considers instead a situation with $b_{2}^{+}$positive for $X$ but zero for $Y$, there will be fixed points consisting of solutions with $M=0$ on $Y$, and one will get a formula expressing invariants of $X \# Y$ in terms of invariants of $X$ and elementary data concerning $Y$.

\section{Vanishing Theorems}

Some of the main properties of the monopole equations can be understood by means of vanishing theorems. The general strategy in deriving such vanishing theorems is quite standard, but as in section two of [12], some unusual cancellations (required by the Lorentz invariance of the underlying untwisted theory) lead to unusually strong results.

If we set $s=F^{+}-M \bar{M}, k=D M$, then a small calculation gives

$$
\begin{aligned}
\int_{X} d^{4} x \sqrt{g}\left(\frac{1}{2}|s|^{2}+|k|^{2}\right)=\int_{X} d^{4} x \sqrt{g} & \left(\frac{1}{2}\left|F^{+}\right|^{2}+g^{i j} D_{i} M^{A} D_{j} \bar{M}_{A}\right. \\
& \left.+\frac{1}{2}|M|^{4}+\frac{1}{4} R|M|^{2}\right) .
\end{aligned}
$$

Here $g$ is the metric of $X, R$ the scalar curvature, and $d^{4} x \sqrt{g}$ the Riemannian measure. A salient feature here is that a term $F_{A B} M^{A} \bar{M}^{B}$, which appears in either $|s|^{2}$ or $|k|^{2}$, cancels in the sum. This sharpens the implications of the formula, as we will see. One can also consider the effect here of the perturbation in (2.12); the sole effect of this is to replace $\frac{1}{2}|M|^{4}$ in (3.1) by

$$
\int_{X} d^{4} x \sqrt{g}\left(F^{+} \wedge p+\sum_{A, B}\left|\frac{1}{2}\left(M_{A} \bar{M}_{B}+M_{B} \bar{M}_{A}\right)-p_{A B}\right|^{2}\right) .
$$

The second term is non-negative, and the first is simply the intersection pairing

$$
2 \pi c_{1}(L) \cdot[p]
$$

An obvious inference from (3.1) is that if $X$ admits a metric whose scalar curvature is positive, then for such a metric, any solution of the monopole equations must have $M=0$ and $F^{+}=0$. But if $b_{2}{ }^{+}>0$, then 
after a generic small perturbation of the metric (which will preserve the fact that the scalar curvature is positive), there are no abelian solutions of $F^{+}=0$ except flat connections. Therefore, for such manifolds and metrics, a solution of the monopole equations is a flat connection with $M=0$. These too can be eliminated using the perturbation in (2.12). ${ }^{6}$ Hence a four-manifold for which $b_{2}^{+}>0$ and $n_{x} \neq 0$ for some $x$ does not admit a metric of positive scalar curvature. (If $b_{2}^{+}=1$, the statement means that the $n_{x}$ are zero for any such metric.)

We can extend this to determine the possible four-manifolds $X$ with $b_{2}^{+}>0$, some $n_{x} \neq 0$, and a metric of non-negative scalar curvature. ${ }^{7}$ If $X$ obeys those conditions, then for any metric of $R \geq 0$, any basic class $x$ is in $H^{2,-}$ modulo torsion (so that $L$ admits a connection with $F^{+}=0$, enabling (3.1) to vanish); in particular if $x$ is not torsion then $x^{2}<0$. Now consider the effect of the perturbation (2.12). As $x \in H^{2,-},(3.3)$ vanishes; hence if $R \geq 0, R$ must be zero, $M$ must be covariantly constant and $(M \bar{M})^{+}=p$ (from (3.2)). For $M$ covariantly constant, $(M \bar{M})^{+}=p$ implies that $p$ is covariantly constant also; but for all $p \in H^{2,+}$ to be covariantly constant implies that $X$ is Kähler with $b_{2}^{+}=1$ or is hyper-Kähler. Hyper-Kähler metrics certainly have $R=0$, and there are examples of metrics with $R=0$ on Kähler manifolds with $b_{2}^{+}=1$ [22]; some $n_{x}$ are non-zero in the former case and very likely in the latter.

As an example, for a Kähler manifold with $b_{2}^{+} \geq 3$, the canonical divisor $K$ always arises as a basic class, as we will see in section four, so except in the hyper-Kähler case, such manifolds do not admit a metric of nonnegative scalar curvature.

Even if the scalar curvature is not positive, we can get an explicit bound from (3.1) showing that there are only finitely many basic classes. Since

$$
\int_{X} d^{4} x \sqrt{g}\left(\frac{1}{2}|M|^{4}+\frac{1}{4} R|M|^{2}\right) \geq-\frac{1}{32} \int_{X} d^{4} x \sqrt{g} R^{2},
$$

it follows from (3.1), even if we throw away the term $\left|D_{i} M\right|^{2}$, that

$$
\int_{X} d^{4} x \sqrt{g}\left|F^{+}\right|^{2} \leq \frac{1}{16} \int_{X} d^{4} x \sqrt{g} R^{2} .
$$

\footnotetext{
${ }^{6}$ Flat connections can only arise if $c_{1}(L)$ is torsion; in that case, $c_{1}(L) \cdot[p]=0$. The vanishing argument therefore goes through, the modification in (3.1) being that which is indicated in (3.2).

${ }^{7}$ If $b_{2}^{+}=1$, the $n_{x}$ are not all topological invariants, and we interpret the hypothesis to mean that with at least one sign of the perturbation in (2.12), the $n_{x}$ are not all zero for the given metric.
} 
On the other hand, basic classes correspond to line bundles $L$ with $c_{1}(L)^{2}=$ $(2 \chi+3 \sigma) / 4$, or

$$
\frac{1}{(2 \pi)^{2}} \int d^{4} x \sqrt{g}\left(\left|F^{+}\right|^{2}-\left|F^{-}\right|^{2}\right)=\frac{2 \chi+3 \sigma}{4} .
$$

Hence, for a basic class, both $I^{+}=\int d^{4} x \sqrt{g}\left|F^{+}\right|^{2}$ and $I^{-}=\int d^{4} x \sqrt{g}\left|F^{-}\right|^{2}$ are bounded. For a given metric, there are only finitely many isomorphism classes of line bundles admitting connections with given bounds on both $I^{+}$and $I^{-}$, so the set of basic classes is finite. This is a result proved by Kronheimer and Mrowka with their definition of the basic classes.

The basic classes correspond, as indicated in section three, to line bundles on which the moduli space of solutions of the monopole equations is of zero virtual dimension. We can analyze in a similar way components of the moduli space of positive dimension. Line bundles $L$ such that $c_{1}(L)^{2}<(2 \chi+3 \sigma) / 4$ are not of much interest in that connection, since for such line bundles the moduli space has negative virtual dimension and is generically empty. But if $c_{1}(L)^{2}>(2 \chi+3 \sigma) / 4$, then (3.6) is simply replaced by the stronger bound

$$
\frac{1}{(2 \pi)^{2}} \int d^{4} x \sqrt{g}\left(\left|F^{+}\right|^{2}-\left|F^{-}\right|^{2}\right)>\frac{2 \chi+3 \sigma}{4} .
$$

The set of isomorphism classes of line bundles admitting a connection obeying this inequality as well as (3.5) is once again finite. So we conclude that for any given metric on $X$, the set of isomorphism classes of line bundles for which the moduli space is non-empty and of non-negative virtual dimension is finite; for a generic metric on $X$, there are only finitely many non-empty components of the moduli space.

For further consequences of (3.1), we turn to a basic case in the study of four-manifolds: the case that $X$ is Kähler.

\section{Computation On Kähler Manifolds}

If $X$ is Kähler and spin, then $S^{+} \otimes L$ has a decomposition $S^{+} \otimes L \cong$ $\left(K^{1 / 2} \otimes L\right) \oplus\left(K^{-1 / 2} \otimes L\right)$, where $K$ is the canonical bundle and $K^{1 / 2}$ is a square root. If $X$ is Kähler but not spin, then $S^{+} \otimes L$, defined as before, has a similar decomposition where now $K^{1 / 2}$ and $L$ are not defined separately and $K^{1 / 2} \otimes L$ is characterized as a square root of the line bundle $K \otimes L^{2}$. 
We denote the components of $M$ in $K^{1 / 2} \otimes L$ and in $K^{-1 / 2} \otimes L$ as $\alpha$ and $-i \bar{\beta}$, respectively. The equation $F^{+}(A)=M \bar{M}$ can now be decomposed

$$
\begin{aligned}
& F^{2,0}=\alpha \beta \\
& F_{\omega}^{1,1}=-\frac{\omega}{2}\left(|\alpha|^{2}-|\beta|^{2}\right) \\
& F^{0,2}=\bar{\alpha} \bar{\beta} .
\end{aligned}
$$

Here $\omega$ is the Kähler form and $F_{\omega}^{1,1}$ is the $(1,1)$ part of $F^{+}$. (3.1) can be rewritten

$$
\begin{aligned}
\int_{X} d^{4} x \sqrt{g}\left(\frac{1}{2}|s|^{2}+|k|^{2}\right) & \\
=\int_{X} d^{4} x \sqrt{g}( & \frac{1}{2}\left|F^{+}\right|^{2}+g^{i j} D_{i} \bar{\alpha} D_{j} \alpha+g^{i j} D_{i} \bar{\beta} D_{j} \beta \\
& \left.+\frac{1}{2}\left(|\alpha|^{2}+|\beta|^{2}\right)^{2}+\frac{1}{4} R\left(|\alpha|^{2}+|\beta|^{2}\right)\right) .
\end{aligned}
$$

Unless $R \geq 0$, the right hand side of (4.2) is not manifestly non-negative, but the fact that it is equal to the left hand side shows that it is non-negative and zero precisely for solutions of the monopole equations. Consider the operation

$$
\begin{aligned}
& A \rightarrow A \\
& \alpha \rightarrow \alpha \\
& \beta \rightarrow-\beta .
\end{aligned}
$$

This is not a symmetry of the monopole equations. But it is a symmetry of the right hand side of (4.2). Therefore, given a zero of the right hand side of (4.2) - that is, a solution of the monopole equations - the operation (4.3) gives another zero of the right hand side of (4.2) - that is, another solution of the monopole equations. So, though not a symmetry of the monopole equations, the transformation (4.3) maps solutions of those equations to other solutions.

Given that any solution of (4.1) is mapped to another solution by (4.3), it follows that such a solution has

$$
0=F^{2,0}=F^{0,2}=\alpha \beta=\bar{\alpha} \bar{\beta} .
$$

Vanishing of $F^{0,2}$ means that the connection $A$ defines a holomorphic structure on $L$. The basic classes (which are first Chern classes of $L$ 's that are 
such that (4.1) has a solution) are therefore of type $(1,1)$ for any Kähler structure on $X$, a severe constraint.

Vanishing of $\alpha \beta$ means that $\alpha=0$ or $\beta=0$. If $\alpha=0$, then the Dirac equation for $M$ reduces to

$$
\bar{\partial}_{A} \beta=0
$$

where $\bar{\partial}_{A}$ is the $\bar{\partial}$ operator on $L$. Similarly, if $\beta=0$, then the Dirac equation gives

$$
\bar{\partial}_{A} \alpha=0
$$

Knowing that either $\alpha$ or $\beta$ is zero, we can deduce which it is. Integrating the $(1,1)$ part of $(4.1)$ gives

$$
\frac{1}{2 \pi} \int_{X} \omega \wedge F=-\frac{1}{4 \pi} \int_{X} \omega \wedge \omega\left(|\alpha|^{2}-|\beta|^{2}\right) .
$$

The left hand side of (4.7) is a topological invariant which can be interpreted as

$$
J=[\omega] \cdot c_{1}(L)
$$

The condition that there are no non-trivial abelian instantons is that $J$ is non-zero; we only wish to consider metrics for which this is so. If $J<0$, we must have $\alpha \neq 0, \beta=0$, and if $J>0$, we must have $\alpha=0, \beta \neq 0$.

The equation that we have not considered so far is the $(1,1)$ part of (4.1). This equation can be interpreted as follows. Suppose for example that we are in the situation with $\beta=0$. The space of connections $A$ and sections $\alpha$ of $K^{1 / 2} \otimes L$ can be interpreted as a symplectic manifold, the symplectic structure being defined by

$$
\begin{aligned}
\left\langle\delta_{1} A, \delta_{2} A\right\rangle & =\int_{X} \omega \wedge \delta_{1} A \wedge \delta_{2} A \\
\left\langle\delta_{1} \alpha, \delta_{2} \alpha\right\rangle & =\frac{-i}{2} \int_{X} \omega \wedge \omega\left(\delta_{1} \bar{\alpha} \delta_{2} \alpha-\delta_{2} \bar{\alpha} \delta_{1} \alpha\right) .
\end{aligned}
$$

The group of $U(1)$ gauge transformations acts on this symplectic manifold. The moment map $\mu$ for this action is the quantity that appears in the $(1,1)$ equation that we have not yet exploited, that is

$$
\mu \omega=F_{\omega}^{1,1}+\frac{\omega}{2}|\alpha|^{2}
$$


By analogy with many similar problems, setting to zero the moment map and dividing by the group of $U(1)$ gauge transformations should be equivalent to dividing by the complexification of the group of gauge transformations. ${ }^{8}$ In the present case, the complexification of the group of gauge transformations acts by $\alpha \rightarrow t \alpha, \bar{\partial}_{A} \rightarrow t \bar{\partial}_{A} t^{-1}$, where $t$ is a map from $X$ to $\mathbf{C}^{*}$.

Conjugation by $t$ has the effect of identifying any two $A$ 's that define the same complex structure on $L$. This can be done almost uniquely: the ambiguity is that conjugation by a constant $t$ does not change $A$. Of course, a gauge transformation by a constant $t$ rescales $\alpha$ by a constant. The result therefore, for $J<0$, is that the moduli space of solutions of the monopole equations is the moduli space of pairs consisting of a complex structure on $L$ and a non-zero holomorphic section, defined up to scaling, of $K^{1 / 2} \otimes L$. For $J>0$, it is instead $\beta$ that is non-zero, and $K^{1 / 2} \otimes L$ is replaced by $K^{1 / 2} \otimes L^{-1}$.

This result can be stated particularly nicely if $X$ has $b_{1}=0$. Then the complex structure on $L$, assuming that it exists, is unique. The moduli space of solutions of the monopole equations is therefore simply a complex projective space, $\mathbf{P} H^{0}\left(X, K^{1 / 2} \otimes L\right)$ or $\mathbf{P} H^{0}\left(X, K^{1 / 2} \otimes L^{-1}\right)$, depending on the sign of $J$.

\section{Identifying The Basic Classes}

We would now like to identify the basic classes. The above description of the moduli space gives considerable information: basic classes are of the form $x=-2 c_{1}(L)$, where $L$ is such that $J<0$ and $H^{0}\left(X, K^{1 / 2} \otimes L\right)$ is non-empty, or $J>0$ and $H^{0}\left(X, K^{1 / 2} \otimes L^{-1}\right)$ is non-empty. This, however, is not a sharp result.

That is closely related to the fact that $\mathbf{P} H^{0}\left(X, K^{1 / 2} \otimes L^{ \pm 1}\right)$, the moduli spaces found above, very frequently have a dimension bigger than the "generic" dimension of the moduli space as predicted by the index theorem. In fact, Kähler metrics are far from being generic. In case the expected dimension is zero, one would have always $n_{x}>0$ if the moduli spaces behaved "generically" (given the complex orientation, an isolated point on the moduli space would always contribute +1 to $n_{x}$; this is a special case of a discussion below). Since the $n_{x}$ are frequently negative (as in the exam-

\footnotetext{
${ }^{8}$ In such comparisons of symplectic and complex quotients, one usually needs a stability condition on the complex side. In the present case, this is the condition discussed in connection with (4.8).
} 
ples of Kronheimer and Mrowka or equation (2.66) of [4]), moduli spaces of non-generic dimension must appear.

When the moduli space has greater than the generically expected dimension, one can proceed by integrating over the bosonic and fermionic collective coordinates in the path integral. This gives a result that can be described topologically: letting $T$ be the operator that arises in linearizing the monopole equations, the cokernel of $T$ is a vector bundle $V$ (the "bundle of antighost zero modes") over the moduli space $\mathcal{M}$; its Euler class integrated over $\mathcal{M}$ is the desired $n_{x}$.

Alternatively, one can perturb the equations to more generic ones. We use the same perturbation as before. For a Kähler manifold $X$, the condition $b_{2}^{+}>1$ is equivalent to $H^{2,0}(X) \neq 0$, so we can pick a non-zero holomorphic two-form $\eta .{ }^{9}$ We perturb the monopole equations (4.1) to

$$
\begin{aligned}
& F^{2,0}=\alpha \beta-\eta \\
& F_{\omega}^{1,1}=\frac{-\omega}{2}\left(|\alpha|^{2}-|\beta|^{2}\right) \\
& F^{0,2}=\bar{\alpha} \bar{\beta}-\bar{\eta},
\end{aligned}
$$

leaving unchanged the Dirac equation for $M$.

It suffices to consider the case that the first Chern class of $L$ is of type $(1,1)$, since the unperturbed moduli space vanishes otherwise. That being so, we have

$$
0=\int_{X} F^{2,0} \wedge \bar{\eta}=\int_{X} F^{0,2} \wedge \eta
$$

Using this, one finds that (4.2) generalizes to

$$
\begin{aligned}
& \int_{X} d^{4} x \sqrt{g}\left(\frac{1}{2}|s|^{2}+|k|^{2}\right) \\
&=\int_{X} d^{4} x\left(\frac{1}{2}\left|F^{+}\right|^{2}+g^{i j} D_{i} \bar{\alpha} D_{j} \alpha+g^{i j} D_{i} \bar{\beta} D_{j} \beta\right. \\
&\left.\quad+\frac{1}{2}\left(|\alpha|^{2}-|\beta|^{2}\right)^{2}+2|\alpha \beta-\eta|^{2}+\frac{R}{4}\left(|\alpha|^{2}+|\beta|^{2}\right)\right) .
\end{aligned}
$$

\footnotetext{
${ }^{9}$ In [4], where essentially the same perturbation was made, the two-form was called $\omega$, but here we reserve that name for the Kähler form.
} 
We can now make an argument of a sort we have already seen: the transformation

$$
\begin{aligned}
& A \rightarrow A \\
& \alpha \rightarrow \alpha \\
& \beta \rightarrow-\beta \\
& \eta \rightarrow-\eta
\end{aligned}
$$

though not a symmetry of (4.11), is a symmetry of the right hand side of (4.13). As solutions of (4.11) are the same as zeroes of the right hand side of (4.13), we deduce that the solutions of (4.11) with a two-form $\eta$ are transformed by (4.14) to the solutions with $-\eta$. The terms in (4.11), even or odd under the transformation, must therefore separately vanish, so any solution of (4.11) has

$$
0=F^{0,2}=F^{2,0}=\alpha \beta-\eta
$$

The condition $F^{0,2}=0$ means that the connection still defines a holomorphic structure on $L$.

The condition

$$
\alpha \beta=\eta
$$

gives our final criterion for determining the basic classes: they are of the form $x=-2 c_{1}(L)$ where, for any choice of $\eta \in H^{0}(X, K)$, one has a factorization $\eta=\alpha \beta$ with holomorphic sections $\alpha$ and $\beta$ of $K^{1 / 2} \otimes L^{ \pm 1}$, and $x^{2}=c_{1}(K)^{2}$.

To make this completely explicit, suppose the divisor of $\eta$ is a union of irreducible components $C_{i}$ of multiplicity $r_{i}$. Thus the canonical divisor is

$$
c_{1}(K)=\sum_{i} r_{i}\left[C_{i}\right]
$$

where $\left[C_{i}\right]$ denotes the cohomology class that is Poincaré dual to the curve $C_{i}$. The existence of the factorization $\eta=\alpha \beta$ means that the divisor of $K^{1 / 2} \otimes L$ is

$$
c_{1}\left(K^{1 / 2} \otimes L\right)=\sum_{i} s_{i}\left[C_{i}\right]
$$


where $s_{i}$ are integers with $0 \leq s_{i} \leq r_{i}$. The first Chern class of $L$ is therefore

$$
c_{1}(L)=\sum_{i}\left(s_{i}-\frac{1}{2} r_{i}\right)\left[C_{i}\right]
$$

And the basic classes are of the form $x=-2 c_{1}(L)$ or

$$
x=-\sum_{i}\left(2 s_{i}-r_{i}\right)\left[C_{i}\right]
$$

An $x$ of this form is of type $(1,1)$ and congruent to $c_{1}(K)$ modulo two, but may not obey $x^{2}=c_{1}(K)^{2}$. It is actually possible to prove using the Hodge index theorem that for $x$ as above, $x^{2} \leq c_{1}(K)^{2} .{ }^{10}$ This is clear from the monopole equations: perturbed to $\eta \neq 0$, these equations have at most isolated solutions (from the isolated factorization $\eta=\alpha \beta$ ) and not a moduli space of solutions of positive dimension. So for Kähler manifolds, the nonempty perturbed moduli spaces are at most of dimension zero; invariants associated with monopole moduli spaces of higher dimension vanish.

Our final conclusion about the basic classes, then, is that they are classes of the form (4.20) such that $x^{2}=c_{1}(K)^{2}$. Each factorization $\eta=\alpha \beta$ contributes \pm 1 to $n_{x}$ with the corresponding $x$. Since several factorizations might give the same $x$, cancellations may be possible, making it possible to write the invariant in the Kronheimer-Mrowka form, with a shorter list of basic classes. Such cancellations can be effectively found since the signs of the various contributions are computed below. In any event the classes $x= \pm K$ arise only from $s_{i}=0$ or $s_{i}=r_{i}$, respectively, and so always arise as basic classes with $n_{x}= \pm 1 .^{11}$

\section{Comparison To Previous Results}

Let us compare these statements to previous results. The main case considered in [4] was that in which the $C_{i}$ were disjoint with multiplicities

\footnotetext{
${ }^{10}$ Such an argument was pointed out by D. Morrison.

${ }^{11}$ G. Tian and S.-T. Yau, P. Kronheimer and T. Mrowka, D. Morrison, and R. Friedman and J. Morgan pointed out that it actually follows from these conditions (or related arguments) that if $X$ is a minimal surface of general type, then the only basic classes are $\pm K$ (so that $K$ is a differentiable invariant up to sign). Indeed, according to Lemma 4 in [23], on such a surface, if $K=\mathcal{O}\left(C_{1}\right) \otimes \mathcal{O}\left(C_{2}\right)$ with non-zero effective divisors $C_{1}, C_{2}$, then $C_{1} \cdot C_{2}>0$. This means that a factorization $\eta=\alpha \beta$ with $\alpha, \beta$ sections of $K^{1 / 2} \otimes L^{ \pm 1}$ and $x^{2}=c_{1}(K)^{2}$ implies that $K^{1 / 2} \otimes L^{ \pm 1}$ is trivial with one choice of sign, and hence that $x= \pm c_{1}(K)$.
} 
$r_{i}=1$. The allowed values of the $s_{i}$ are then 0 and 1 , so the basic classes are

$$
x_{\vec{\rho}}=\sum_{i} \rho_{i}\left[C_{i}\right]
$$

with each $\rho_{i}= \pm 1$, as claimed in [4]. Notice that all of these classes have $x_{\vec{\rho}}^{2}=c_{1}(K)^{2}$.

The most important case in which the $r_{i}$ are not all one is the case of an elliptic surface with multiple fibers. A fiber of multiplicity $n$ appears in the canonical divisor with weight $r=n-1$. For elliptic surfaces, one has $C_{i} \cdot C_{j}=0$ for all $i, j$, so all the classes in (4.20) actually do have $x^{2}=c_{1}(K)^{2}=0$. The formulas conjectured by Kronheimer and Mrowka and proved by Fintushel and Stern ${ }^{12}$ for the Donaldson invariants of these surfaces show that the basic classes, in their sense, are indeed the classes given in (4.20).

\section{Determination Of The Sign}

To complete this story, we must compute, for each factorization, the sign of $\operatorname{det} T$. Let us first explain in an abstract setting the strategy that will be used. Suppose that $E$ and $F$ are real vector spaces of equal even dimension with given complex structures, and $T: E \rightarrow F$ is an invertible linear map that commutes with the complex structure. Then $\operatorname{det} T$ is naturally defined as an element of $\operatorname{det} F \otimes \operatorname{det} E^{-1}$. If $\operatorname{det} E$ and $\operatorname{det} F$ are trivialized using the complex orientations of $E$ and $F$, then $\operatorname{det} T>0$ roughly because the complex structure gives a pairing of eigenvalues. If $T$ reverses the complex structures then the sign of $\operatorname{det} T$ is $(-1)^{w}$ with $w=\operatorname{dim}_{\mathbf{C}} E$. For instance, by reversing the complex structure of $E$ one could reduce to the case in which $T$ preserves the complex structures, but reversing the complex structure of $E$ multiplies its orientation by $(-1)^{w}$.

One can combine the two cases as follows. Suppose that $T$ preserves the complex structures but is not invertible. Let $T^{\prime}: E \rightarrow F$ be a map that reverses the complex structures and maps ker $T$ invertibly to $F / T(E)$. Then for small real $\epsilon$ (of any sign) the sign of $\operatorname{det}\left(T \oplus \epsilon T^{\prime}\right)$ is $(-1)^{w}$ where now $w=\operatorname{dim}_{\mathbf{C}} \operatorname{ker} T$. The same formula holds if $U$ and $V$ are vector bundles, $E=\Gamma(U), F=\Gamma(V), T: E \rightarrow F$ is an elliptic operator with zero index, $T^{\prime}$ is a sufficiently mild perturbation, and $\operatorname{det}\left(T+\epsilon T^{\prime}\right)$ is understood as the Ray-Singer-Quillen determinant.

\footnotetext{
${ }^{12}$ R. Fintushel and R. Stern, "Rational blowdowns of smooth 4-manifolds," to appear.
} 
Our problem is of this form with $T$ understood as the linearization of the monopole equations at $\eta=0$ and $T^{\prime}$ as the correction proportional to $\eta$ (which enters the linearization because of the shift it induces in $\alpha$ or $\beta$ ). As in (2.4), one has $U=\Lambda^{1} \oplus\left(S^{+} \otimes L\right)$, with $S^{+} \otimes L$ now regarded as a real vector bundle of rank four. If $J<0$ (so $\beta=0$ for $\eta=0$ ), then give $U$ a complex structure that acts naturally on $S^{+} \otimes L$ and multiplies $\Lambda^{0,1}$ and $\Lambda^{1,0}$ by $i$ and $-i$, respectively. Likewise give $V=\Lambda^{0} \oplus \Lambda^{2,+} \oplus\left(S^{-} \otimes L\right)$ a complex structure that acts naturally on $S^{-} \otimes L$; multiplies $\Lambda^{0,2}$ and $\Lambda^{2,0}$ by $i$ and $-i$; and exchanges the $(1,1)$ part of $\Lambda^{2,+}$ with $\Lambda^{0}$. Then $T$ preserves the complex structures on these bundles and $T^{\prime}$ reverses them.

The sign of the contribution to $n_{x}$ from a factorization $\eta=\alpha \beta$ is therefore $(-1)^{w}$ with $w=\operatorname{dim}_{\mathbf{C}} \operatorname{ker} T$. The kernel of $T$ can be described as follows. There is an exact sequence

$$
0 \rightarrow \mathcal{O} \stackrel{\alpha}{\longrightarrow} K^{1 / 2} \otimes L \rightarrow R \rightarrow 0
$$

with some sheaf $R$. The kernel of $T$ has the same dimension as $H^{0}(X, R)$, as explained below. So the sign of the contribution to $n_{x}$ is

$$
(-1)^{\operatorname{dim} H^{0}(X, R)} \text {. }
$$

If instead $J>0$, so the unperturbed solution has $\alpha=0, \beta \neq 0$, then first of all, we reverse the complex structures on $S^{ \pm} \otimes L$; this multiplies the determinant by $(-1)^{\Delta}$ where $\Delta=-\sigma / 8+c_{1}(L)^{2} / 2=(\chi+\sigma) / 4$ is the Dirac index. The rest of the discussion goes through with (4.22) replaced by

$$
0 \rightarrow \mathcal{O} \stackrel{\beta}{\longrightarrow} K^{1 / 2} \otimes L^{-1} \rightarrow \widetilde{R} \rightarrow 0
$$

so the sign is

$$
(-1)^{\Delta+\operatorname{dim} H^{0}(X, R)}
$$

(It can be verified using the classification of surfaces that (4.23) and (4.25) are equal.) With these signs, (2.14) becomes completely explicit: the sum in (2.14) is a sum over factorizations $\eta=\alpha \beta$; each such factorization determines a class $x$ and contributes to $n_{x}$ an amount \pm 1 as just determined.

Before justifying the claim about $\operatorname{ker} T$, let us check that the sign just determined agrees with what has been computed by other methods. Suppose as in [4] that the divisor of $\eta$ is a union of disjoint smooth curves $C_{i}$ 
of multiplicity one. Then $R$ is a sum of sheaves $R_{i}$ supported on $C_{i} ; R_{i}$ is trivial if $s_{i}$ (defined in (4.18)) is 0 and is isomorphic to a spin bundle of $C_{i}$ (determined by $\eta$ and independent of the factorization $\eta=\alpha \beta$ ) if $s_{i}=1$. Let $t_{i}=1$ if this spin bundle is even, that is, if $\operatorname{dim} H^{0}\left(C_{i}, R_{i}\right)$ is even, and -1 if it is odd. Then (4.23) becomes

$$
(-1)^{\operatorname{dim} H^{0}(X, R)}=\prod_{i \mid s_{i}=1} t_{i} .
$$

This is the result claimed in equation (2.66) of [4]. One can similarly check that (2.14) when evaluated with the signs given above, agrees with the known formulas for Donaldson invariants of elliptic surfaces with multiple fibers.

It remains to justify the claimed structure of ker $T$. Suppose, for instance, that we are linearizing around a solution with $\beta=0, \alpha \neq 0$. Let $\delta A, \delta \alpha$, and $\delta \beta$ denote first order variations of $A, \alpha$, and $\beta$. The argument that proves the vanishing theorem shows that for $\delta A, \delta \alpha$, and $\delta \beta$ to be annihilated by $T$, one must have $\alpha \delta \beta=0$ and hence $\delta \beta=0$. The remaining equations can be written

$$
\begin{array}{r}
\bar{\partial} \delta A^{0,1}=0 \\
i \delta A^{0,1} \alpha+\bar{\partial}_{A} \delta \alpha=0 .
\end{array}
$$

One must divide the space of solutions of (4.27) by solutions that arise from complex gauge transformations of $A, \alpha$. If $\delta A^{0,1}=0$, then the second equation says that $\delta \alpha \in H^{0}\left(X, K^{1 / 2} \otimes L\right)$; however, upon dividing by complex gauge transformations (which include rescalings of $\alpha$ by a constant) we should regard $\delta \alpha$ as an element of $H^{0}\left(X, K^{1 / 2} \otimes L\right) / \mathbf{C} \alpha$. The first equation says that $\delta A^{0,1}$ defines an element of $H^{1}(X, \mathcal{O})$, and the second equation says that multiplication by $\alpha$ maps this element to zero in $H^{1}\left(X, K^{1 / 2} \otimes L\right)$. So if ker $\alpha$ is the kernel of $H^{1}(X, \mathcal{O}) \stackrel{\alpha}{\longrightarrow} H^{1}\left(X, K^{1 / 2} \otimes L\right)$, then there is an exact sequence

$$
0 \rightarrow H^{0}\left(X, K^{1 / 2} \otimes L\right) / \mathbf{C} \alpha \rightarrow \operatorname{ker} T \rightarrow \operatorname{ker} \alpha \rightarrow 0 .
$$

This can be compared to the exact sequence

$$
0 \rightarrow H^{0}\left(X, K^{1 / 2} \otimes L\right) / \mathbf{C} \alpha \rightarrow H^{0}(X, R) \rightarrow \operatorname{ker} \alpha \rightarrow 0
$$

that comes from (4.22). Comparison of these sequences shows that $\operatorname{ker} T$ and $H^{0}(X, R)$ have the same dimension, as asserted above; one should be able to identify these spaces canonically. 


\section{A Short Sketch Of The Physics}

To sketch the relation of these ideas to quantum field theory, let us first recall the analysis of $N=2$ supersymmetric Yang-Mills theory in $[5,6]$. To begin, we work on flat $\mathbf{R}^{4}$. It has long been known that this theory has a family of quantum vacuum states parametrized by a complex variable $u$, which corresponds to the four dimensional class in Donaldson theory. For $u \rightarrow \infty$, the gauge group is spontaneously broken down to the maximal torus, the effective coupling is small, and everything can be computed using asymptotic freedom. For small $u$, the effective coupling is strong. Classically, at $u=0$, the full $S U(2)$ gauge symmetry is restored. But the classical approximation is not valid near $u=0$.

Quantum mechanically, as explained in $[5,6]$, the $u$ plane turns out to parametrize a family of elliptic curves, ${ }^{13}$ in fact, the modular curve of the group $\Gamma(2)$. The family can be described by the equation

$$
y^{2}=\left(x^{2}-\Lambda^{4}\right)(x-u)
$$

where $\Lambda$ is the analog of a parameter that often goes by the same name in the theory of strong interactions. (The fact that $\Lambda \neq 0$ means that the quantum theory does not have the conformal invariance of the classical theory.) The curve (5.1) is smooth for generic $u$, but degenerates to a rational curve for $u=\Lambda^{2},-\Lambda^{2}$, or $\infty$. Near each degeneration, the theory becomes weakly coupled, and everything is calculable, if the right variables are used. At $u=\infty$, the weak coupling is (by asymptotic freedom) in terms of the original field variables. Near $u= \pm \Lambda^{2}$, a magnetic monopole (or a dyon, that is, a particle carrying both electric and magnetic charge) becomes massless; the light degrees of freedom are the monopole or dyon and a dual photon or $U(1)$ gauge boson. In terms of the dyon and dual photon, the theory is weakly coupled and controllable near $u= \pm \Lambda^{2}$.

Notice that quantum mechanically on flat $\mathbf{R}^{4}$, the full $S U(2)$ gauge symmetry is never restored. The only really exceptional behavior that occurs anywhere is that magnetically charged particles become massless.

\footnotetext{
${ }^{13}$ If $S U(2)$ is replaced by a Lie group of rank $r$, elliptic curves are replaced by abelian varieties of rank $r$; the analog of the simple type condition is that the commutative algebra of operators obtained by evaluating the Chern classes of the universal bundle at a point in a four-manifold has a spectrum consisting of $h$ points ( $h$, which is $N$ for $S U(N)$, is the dual Coxeter number of the Lie group) where these varieties degenerate maximally.
} 
Now, for any $N=2$ supersymmetric field theory, a standard twisting procedure [1] gives a topological field theory. In many cases, these topological field theories are related to the counting of solutions of appropriate equations. For instance, the procedure, applied to the underlying $S U(2)$ gauge theory, gives Donaldson theory (that is, the problem of counting $S U(2)$ instantons); applied to the quantum theory near $u= \pm \Lambda^{2}$, it gives the problem of counting the solutions of the monopole equations; applied at a generic point on the $u$ plane, it gives, roughly, the problem of counting abelian instantons.

Now let us apply this experience, to work on a general oriented fourmanifold $X$. The structure of the argument is analogous to the heat kernel proof of the index theorem, in which one considers the trace of the heat kernel $\operatorname{Tr}(-1)^{F} e^{-t H}$. This is independent of $t$ but can be evaluated in different ways for $t \rightarrow 0$ or for $t \rightarrow \infty$; for small $t$, one sees local geometry and gets a cohomological formula, while for large $t$, one gets a description in terms of the physical ground states (harmonic spinors).

In the four-manifold problem, letting $g$ be any Riemannian metric on $X$, we consider the one parameter family of metrics $g_{t}=t g$, with $t>0$. Correlation functions of the twisted topological field theory are metric independent and so independent of $t$. For $t \rightarrow 0$, using asymptotic freedom, the classical description becomes valid, and one recovers Donaldson's definition of four-manifold invariants from the $N=2$ theory. In particular, for four-manifolds on which there are no abelian instantons, the main contribution comes from $u=0$ where for small $t$, one computes in the familiar fashion with the full $S U(2)$ gauge theory.

What happens for large $t$ ? Once the scale of the four-manifold is much greater than $1 / \Lambda$, the good description is in terms of the degrees of freedom of the vacuum states on $\mathbf{R}^{4}$. At first sight, it might appear that the answer will come by integration over the $u$ plane. That is apparently so for some classes of problems.

However, for four-manifolds with $b_{2}^{+}>1$, one can show that the contribution of any region of the $u$ plane bounded away from $u= \pm \Lambda^{2}$ vanishes as a power of $t$ for $t \rightarrow \infty$. This is roughly because in the abelian theory that prevails away from $u= \pm \Lambda^{2}$, there are too many fermion zero modes and no sufficiently efficient way to lift them.

Under the above condition on $b_{2}^{+}$, a contribution that survives for $t \rightarrow \infty$ can therefore come only from a neighborhood of $u= \pm \Lambda^{2}$ that shrinks to zero as $t$ grows. The contribution from this region does survive for $t \rightarrow \infty$; it 
can be computed using the monopole equations since those are the relevant equations in the topologically twisted theory near $u= \pm \Lambda^{2}$. In computing a correlation function of operators of the twisted theory near $u= \pm \Lambda^{2}$, one can expand all operators of the microscopic theory in terms of operators of successively higher dimension in the macroscopic, monopole theory.

For $u$, the most relevant term (that is, the term of lowest dimension) is the $c$-number $u=\Lambda^{2}$ or $u=-\Lambda^{2}$. The simple type condition-which asserts that $u$ is semi-simple with a spectrum consisting of two pointsarises when one may replace $u$ by this $c$-number. For the operator related to the two-dimensional classes of Donaldson theory, the most relevant term is again a $c$-number, measuring the first Chern class of the dual line bundle $L$ of the monopole problem. Keeping only these terms, since the operators are replaced by $c$-numbers, correlation functions can be computed by simply counting solutions weighted by the sign of the fermion determinant; only zero dimensional moduli spaces contribute. Upon fixing the normalizations by comparing to known special cases, one arrives at (2.14).

However, higher terms in the expansion of the operators can survive when the higher monopole invariants of $W \neq 0$ are non-zero. Then (2.14) will be replaced by a more general formula involving the expansion near $u= \pm \Lambda^{2}$ of some of the functions computed in $[5,6]$. The number $s$ of higher terms that one would have to keep in the expansion would be one half the maximum value of $W$ that contributes. $u$ will still have a spectrum consisting of two points, but instead of $u^{2}-\Lambda^{4}=0$, one would get $\left(u^{2}-\right.$ $\left.\Lambda^{4}\right)^{s+1}=0$. Such a situation has in fact been analyzed by Kronheimer and Mrowka.

\section{References}

1. E. Witten, Topological quantum field theory, Commun. Math. Phys. 117 (1988), 353-386.

2. S. Donaldson, Polynomial invariants for smooth four-manifolds, Topology, 29 (1990) 257-315.

3. A. Floer, An Instanton invariant for 3-manifolds, Commun. Math. Phys. 118 (1988), 215-240.

4. E. Witten, Supersymmetric Yang-Mills theory on a four-manifold, J. Math. Phys. 35 (1994), 5101-5135.

5. N. Seiberg and E. Witten, Electric-magnetic duality, monopole condensation, and confinement in $N=2$ supersymmetric Yang-Mills theory, Nucl. Phys. B426 (1994), 19.

6. Monopoles, duality, and chiral symmetry breaking in $N=2$ supersymmetric $\overline{Q C D}$, hep-th/9408099, to appear in Nucl. Phys. B. 
7. N. Seiberg, The power of holomorphy-exact results in $4 d$ SUSY field theories, hepth/9408013.

8. P. Kronheimer and T. Mrowka, Recurrence relations and asymptotics for four-manifold invariants, Bull. Am. Math. Soc. 30 (1994), 215-221.

9. __ Embedded surfaces and the structure of Donaldson's polynomial invariants, preprint (1994).

10. P. Argyres and A. Faraggi, The vacuum structure and spectrum of $N=2$ supersymmetric $S U(N)$ gauge theory, hep-th/9411057.

11. A. Klemm, W. Lerche, S. Yankielowicz, and S. Theisen, Simple singularities and $N=2$ supersymmetric Yang-Mills theory, hep-th/9411048.

12. C. Vafa and E. Witten, A strong coupling test of $S$-duality, hep-th/9408074, to appear in Nucl. Phys. B.

13. C. Montonen and D. Olive, Gauge theories and magnetic charge, Phys. Lett. B72 (1977), 117-120.

14. P. Goddard, J. Nuyts, and D. Olive, Magnetic monopoles as gauge particles, Nucl. Phys. B125 (1977), 1-28.

15. A. Sen, Strong-weak coupling duality in 4 dimensional string theory, hep-th/9402002.

16. N. Seiberg and E. Witten, to appear.

17. F. Hirzebruch and H. Hopf, Felder von Flachenelementen in 4-dimensionalen Mannigfaltigkeiten, Math. Annalen 136 (1958), 156-172.

18. S. Donaldson, The orientation of Yang-Mills moduli spaces and four-manifold topology, J. Diff. Geom. 26 (1987), 397-428.

19. K. Uhlenbeck, Removable singularities in Yang-Mills fields, Commun. Math. Phys. 83 (1982), 11-29.

20. C. H. Taubes, Self-dual Yang-Mills connections over non-self-dual 4-manifolds, J. Diff. Geom. 19 (1982), 517-560.

21. S. Donaldson, Irrationality and the h-cobordism conjecture, J. Diff. Geom. 26 (1987), 141-168.

22. C. LeBrun, Scalar-flat Kähler metrics on blown-up ruled surfaces, J. Reine Angew Math. 420 (1991), 161-177.

23. K. Kodaira, Pluricanonical systems on algebraic surfaces of general type, J. Math. Soc. Japan 20 (1968), 170-192.

School of Natural Sciences, Institute for Advanced Study, Olden Lane,

PRINCETON, N J 08540

E-mail address: witten@sns.ias.edu 